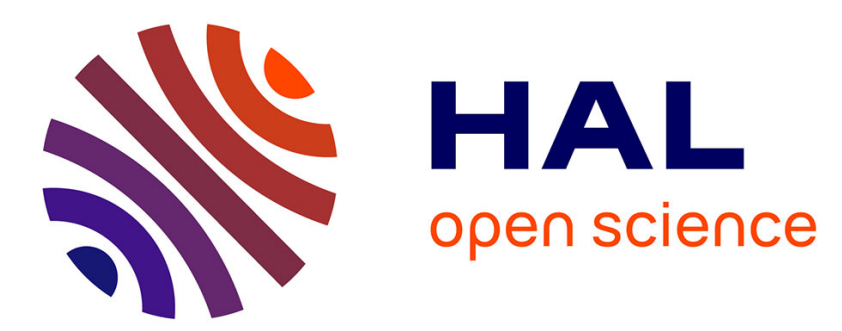

\title{
Evaluation de CORNGRO : un modèle de simulation des transferts hydriques et de la croissance d'une culture de maïs
}

\author{
Marc Voltz, S. Rambal
}

\section{- To cite this version:}

Marc Voltz, S. Rambal. Evaluation de CORNGRO : un modèle de simulation des transferts hydriques et de la croissance d'une culture de maïs. Agronomie, 1987, 7 (9), pp.727-738. hal-00885047

\section{HAL Id: hal-00885047 https://hal.science/hal-00885047}

Submitted on 1 Jan 1987

HAL is a multi-disciplinary open access archive for the deposit and dissemination of scientific research documents, whether they are published or not. The documents may come from teaching and research institutions in France or abroad, or from public or private research centers.
L'archive ouverte pluridisciplinaire HAL, est destinée au dépôt et à la diffusion de documents scientifiques de niveau recherche, publiés ou non, émanant des établissements d'enseignement et de recherche français ou étrangers, des laboratoires publics ou privés. 


\title{
Evaluation de CORNGRO : un modèle de simu- lation des transferts hydriques et de la croissance d'une culture de maïs
}

\author{
Marc VOLTZ \& S. RAMBAL* \\ I.N.R.A., Station de Science du Sol, Centre de Recherches de Montpellier, 34060 Montpellier Cedex \\ * CEPE-CNRS, 34033 Montpellier Cedex, France
}

\begin{abstract}
CORNGRO utilise des entrées climatiques journalières : températures de l'air maximale et minimale, évapotranspiration potentielle, rayonnement global et précipitations. Il simule les flux hydriques dans le système solplante. Le sol est caractérisé par la conductivité hydraulique et le potentiel de pression en fonction de la teneur en eau. Le modèle de croissance répartit la production végétale en quatre compartiments : feuilles, tiges, racines et épis. Le taux de photosynthèse au point de saturation à la lumière et le coefficient qui lie l'accroissement de la matière sèche foliaire à celui de la surface des feuilles sont déterminés par calage. La répétition de cette procédure en année sèche et en année humide permet d'évaluer la capacité prédictive du modèle. L'analyse de sensibilité nous renseigne sur la précision nécessaire de certains paramètres. Les variations de la relation potentiel de pression-teneur en eau, des sommes de température et de la profondeur racinaire, affectent significativement les simulations. Le modèle est relativement insensible aux variations de la distance interracinaire et à la relation conductivité hydraulique-teneur en eau.
\end{abstract}

Mots clés additionnels : Calage du modèle, analyse de sensibilité.

\begin{abstract}
Analysis of CORNGRO : a simulation model of corn growth and water balance.
CORNGRO requires daily environmental inputs : maximum and minimum air temperature, potential evapotranspiration, incoming solar radiation and rainfall. It simulates water flow in the soil-plant system. Soil properties needed are hydraulic conductivity and soil water potential as functions of water content. The growth model shares plant production between four compartments : leaves and stem, roots and ears. The photosynthetic rate at high radiation intensity and the coefficient which relates leaf dry matter accumulation and leaf area growth are set by calibration. Repeating this procedure for wet and dry years allows one to test the predictive ability of the model. A sensitivity analysis was performed to indicate the required accuracy of certain parameters. Variations in soil water potential function, degree-day accumulation and root depth significantly affected model simulations. The model was relatively insensitive to changes of distance between roots and soil hydraulic conductivity function.
\end{abstract}

Additional key words : Model calibration, sensitivity analysis.

\section{INTRODUCTION}

L'estimation de la consommation en eau des cultures et de son efficience en terme de production végétale est une étape indispensable en vue d'une optimisation de l'irrigation. Dans cette optique, des modèles couplés de simulation du bilan hydrique et de la croissance d'une culture sur toute sa période de développement ont été développés. La complexité de ces modèles est très variable. Ainsi certains modèles de croissance consistent en une relation empirique entre le rendement et l'évapotranspiration ou la transpiration (FEDDES et al., 1978 ; VAN KEULEN, 1982) tandis que d'autres tentent une description des mécanismes physiologiques et morphologiques de l'accumulation de matière sèche (DE WIT, 1978; CHILDS et al., 1977 ; STAPPER \& ARKIN, 1980). Parallèlement la simulation du bilan hydrique est effectuée, soit par des modèles «à réservoirs 》 (RAMBAL \& CORNET, 1982 ; VAN KEULEN, 1982), soit par des modèles décrivant les flux hydriques transitoires en zone non saturée du sol (NIMAH \& HANKS, 1973 ; FEDDES et al., 1978 ; CHILDS et al., 1977). Le niveau de description du système sol-plante de ces modèles est donc très hétérogène, ils diffèrent en conséquence par leur nombre de variables et paramètres d'entrée et par l'étendue des conditions aux limites possibles. Notons cependant que ces modèles présentent généralement deux caractéristiques permanentes. La première est de tenir compte des variations de surface foliaire d'une culture au cours de son développement, afin de simuler la partition de l'évapotranspiration en évaporation et transpiration. La seconde est de rendre compte de l'influence de la contrainte hydrique sur la croissance de la culture. 
De nombreux modèles de croissance de culture de maïs ont déjà été conçus. Il s'agit notamment des modèles de CURRY \& CHEN (1971), SIMAIZ de DUNCAN (1975), CORMOD de BAKER \& HORROCKS (1976), CORNGRO de CHILDS et al. (1977), CORNF de STAPPER \& ARKIN (1980), de ceux de GARCIA de CORTAZAR (1982) et de STOCKLE \& CAMPBELL (1985), et plus récemment du modèle CERES de JONES \& KINIRY (1986). Lés potentialités d'utilisation courante de tels modèles ont rarement été explorées. Seuls CORNGRO et CORNF ont donné lieu à des simulations, menées dans un grand nombre de situations climatiques et édaphiques et pour de nombreuses variétés.

On se propose dans cet article d'étudier les principes et les domaines d'utilisation d'un modèle couplé de simulation des transferts hydriques et de la production de matière sèche d'une culture de maïs. Parmi les modèles existants nous avons concentré notre étude sur CORNGRO (CHILDS et al., 1977).

Ce modèle a déjà fait l'objet de plusieurs contrôles expérimentaux; il présente également l'avantage d'être applicable dans de nombreuses conditions pédologiques, du fait d'une représentation élaborée des transferts en zone non saturée du sol. CHILDS et al. (1977), ainsi que TSCHESCHKE \& GILLEY (1979) montrent que pour une année, un site et une variété donnés, CORNGRO estime de manière fiable le rendement, la surface foliaire et les termes du bilan hydrique pour des doses d'irrigation très variables. Le fonctionnement du modèle repose néanmoins sur des coefficients de calage, qui devront être réestimés pour d'autres conditions d'environnement et d'autres variétés. Les validations expérimentales de CORNGRO, que présentent ces auteurs, ont été obtenues dans des conditions locales. Elles ne sont donc pas suffisantes pour fonder l'extrapolation du modèle à d'autres conditions environnementales. Pour compléter les enseignements acquis par des expérimentations de validation locale, des tests numériques du modèle permet- tent de mieux définir ses principes élémentaires de mise en œuvre et de rechercher ses limites de validité. Aussi après avoir présenté une description succincte du modèle, nous analyserons numériquement le rôle et l'estimation des coefficients de calage. Ceux-ci ont au départ une signification physiologique, mais, par le rôle qu'ils jouent, ils intègrent une partie de la variance non expliquée par le modèle et perdent leur sens d'origine. Nous estimerons ensuite par la technique de l'analyse de sensibilité, l'importance de chaque paramètre du modèle et la précision souhaitable de sa mesure en vue de l'utilisation de CORNGRO.

\section{DESCRIPTION DU MODÈLE CORNGRO}

CORNGRO a été conçu pour simuler la croissance des cultures de maïs en fonction de certaines variables de leur environnement. La version initiale du modèle a été décrite par CHILDS et al. (1977). TSCHESCHKE \& GILLEY (1979) présentent une version améliorée qui constitue ici notre matériel d'étude. Le lecteur à la recherche d'une description détaillée de CORNGRO, consultera CHILDS et al., 1977 ; TSCHESCHKE \& GILLEY, 1979 ; KUNDU et al., 1982 ; VOLTZ, 1982.

CORNGRO se décompose en deux sous-modèles interdépendants : l'un simule les transferts d'eau dans le système sol-plante et l'autre la croissance du maïs. Le diagramme de flux de la figure 1 montre les relations entre les différents processus élémentaires pris en compte dans ce modèle. Les variables qui décrivent l'état du système à un instant donné (inscrites dans des rectangles) sont calculées à partir de :

- flux de matière (lignes continues), contrôlées par des valves,

- flux d'informations (lignes pointillées) provenant de variables auxiliaires (cercles).

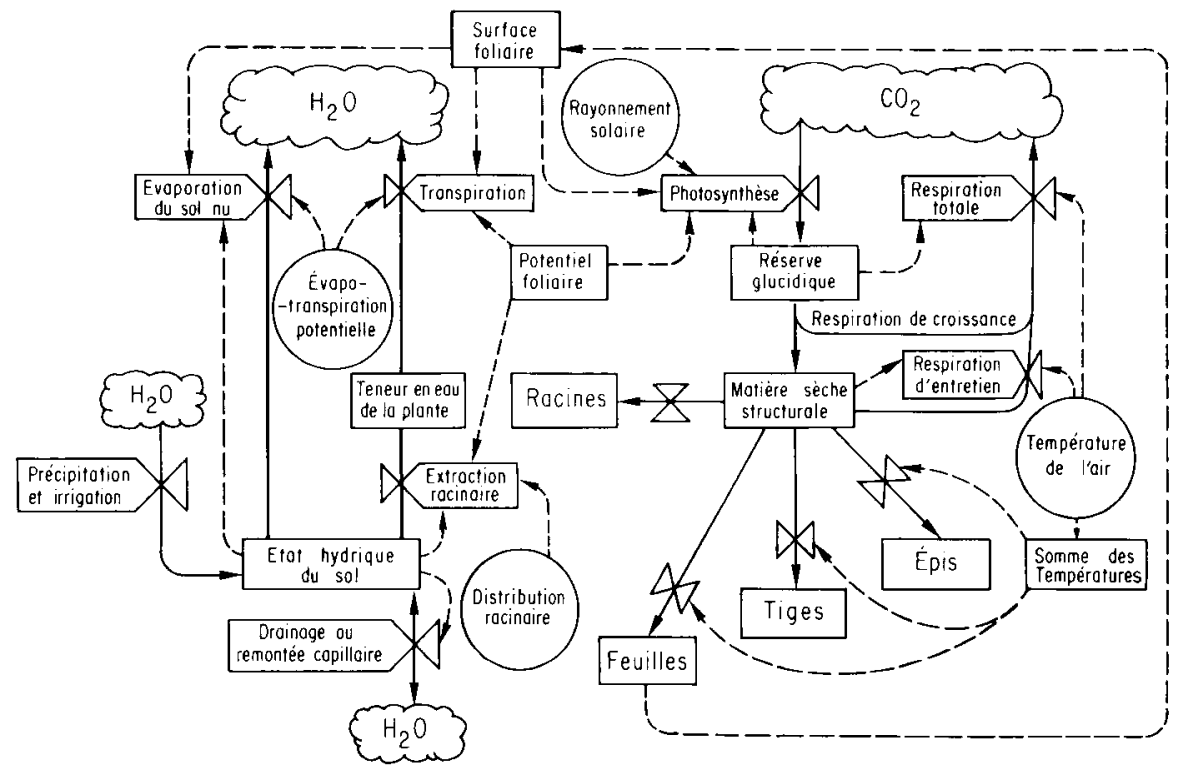

Diagramme des flux du modèle CORNGRO. Les rectangles repré sentent les variables d'état, les lignes continues et tiretées indiquent respectivement les flux de matière et d'information et les valves symbolisent les processus qui influencent ces flux.
Flow diagram of the CORNGRO model. Rectangles are state variables, solid and dashed lines indicate flow of materials and information respectively and valves represent processes influencing flows. 


\section{A. Le modèle des transferts d'eau}

\section{Les transferts d'eau dans le sol}

L'écoulement de l'eau dans le sol, considéré comme un milieu poreux, isotherme et rigide, est décrit par la loi de Darcy généralisée, associée à une équation de continuité qui tient compte de l'extraction de l'eau par les racines. Cette équation est résolue à l'aide d'un schéma numérique aux différences finies. Les conditions aux limites à la partie supérieure du profil de sol sont imposées par les flux d'évaporation et d'infiltration. A la partie inférieure, l'utilisateur choisit une condition de flux ou une condition de potentiel selon le cas considéré. Le terme d'extraction racinaire $S(z)$ est tel que l'extraction racinaire cumulée sur toute la profondeur racinaire $\mathrm{z}^{*}$ soit égale à la transpiration de la culture.

\section{Evaporation potentielle et transpiration potentielle}

L'évaporation à partir du sol nu et la transpiration de la végétation sont considérées comme 2 processus disjoints. L'évaporation potentielle du sol nu, en $\mathrm{cm} \cdot \mathrm{h}^{-1}$, est estimée à partir de l'évapotranspiration potentielle ETP, en faisant l'hypothèse d'une décroissance exponentielle de cette dernière au sein du couvert végétal.

$$
E P=E T P e^{-\beta i F}
$$

iF est l'indice foliaire de la culture, le coefficient d'extinction $\beta$ est pris égal à 0,438 .

La part d'évapotranspiration potentielle non utilisée par l'évaporation potentielle du sol nu fixe la borne supérieure de la transpiration ou transpiration potentielle TRP.

\section{Evaporation réelle et infiltration}

Le flux d'évaporation réelle ou celui d'infiltration est calculé en appliquant la loi de Darcy généralisée à la première couche du sol, selon la procédure utilisée par NiMAH \& HANKS (1973). Le flux est de signe négatif pour l'évaporation, il est positif pour l'infiltration. Lors d'un épisode pluvieux ou d'une irrigation, l'ETP horaire est directement retranchée de l'intensité de l'apport d'eau.

\section{Transpiration réelle et extraction racinaire}

Le transfert de l'eau dans la plante est décomposé en 2 étapes. L'une correspond au transfert de l'eau du sol jusqu'au collet de la plante à travers les racines. Le collet est ici la partie de la plante qui se situe au niveau de la surface du sol. La seconde étape concerne le transfert de l'eau du collet jusqu'aux feuilles. Pour chacune de ces étapes, l'équation qui régit le transfert est bâtie suivant les hypothèses traditionnelles de l'analogie électrique et d'un flux conservatif. La transpiration horaire TR est égale à la différence de potentiel entre les feuilles et le collet $\left(\mathrm{h}_{\mathrm{f}}-\mathrm{h}_{\mathrm{c}}\right)$, divisée par la résistance du xylème des parties aériennes $\left(r_{x}\right)$.

Pour satisfaire la transpiration TR, les racines extraient une certaine quantité d'eau de chaque pro- fondeur du sol. L'extraction racinaire dans une couche d'épaisseur $\Delta \mathrm{z}$ à une profondeur $\mathrm{z}<\mathrm{z}^{*}$ est décrite par l'équation de NIMAH \& HANKS (1973) :

$$
S(z)=\frac{h_{c}-h+z}{r_{s}(z) \cdot \Delta z} \quad \text { si } \quad h_{c}<h
$$

ou $\quad \mathrm{S}(\mathrm{z})=0 \quad$ si $\mathrm{h}_{\mathrm{c}}>\mathrm{h}$

$\mathrm{h}$ représente le potentiel de pression à la cote $\mathrm{z}$, en $\mathrm{cm}$. $\mathrm{r}_{\mathrm{s}}$ constitue la résistance hyraulique de l'interface solracine, en heures :

$$
\mathrm{r}_{\mathrm{s}}(\mathrm{z})=\frac{\Delta \mathrm{x}}{\operatorname{RDF}(\mathrm{z}) \cdot \mathrm{k}(\mathrm{z})}
$$

$\Delta \mathrm{x}$ est la distance moyenne entre deux racines, en $\mathrm{cm}$. $\mathrm{RDF}(\mathrm{z})$ est la densité relative des racines à la cote $\mathrm{z}$, et $\mathrm{k}$ est la conductivité hydraulique du sol à cette même cote. $h_{f}$ et $h_{c}$ sont estimés de manière à rester supérieurs à - 16,5 bars et à assurer un flux de transpiration maximal mais néanmoins inférieur à la transpiration potentielle TRP.

\section{B. Le modèle de croissance du maïs}

Le processus de production végétale est envisagé comme un simple bilan horaire entre les apports des produits bruts de la photosynthèse, le stockage des glucides de réserve et les pertes par respiration.

$$
\Delta \mathrm{S}=\mathrm{P}-\mathrm{R}_{\mathrm{t}}-\Delta \mathrm{G}
$$

$\Delta S$ et $\Delta G$ sont les variations horaires de la fraction structurale et de la réserve glucidique, $P$ est la photosynthèse brute horaire et $R_{t}$ la respiration totale horaire. Toutes ces variables sont exprimées en mg $\mathrm{CH}_{2} \mathrm{O} . \mathrm{dm}^{-2}$ feuille.

\section{Photosynthèse brute horaire}

La photosynthèse brute est calculée par un modèle empirique des facteurs limitants.

$$
P=f_{1} \cdot f_{2} \cdot f_{3} \cdot f_{4} \cdot P_{0}
$$

$\mathrm{P}_{0}$ en $\mathrm{mg} \mathrm{CH}_{2} \mathrm{O} \cdot \mathrm{dm}^{-2}$ feuille. $\mathrm{h}^{-1}$ représente la photosynthèse brute du couvert au point de saturation à la lumière, ici pour un éclairement voisin de $1000 \mathrm{~W} . \mathrm{m}^{-2}$ et en l'absence de toute autre limitation. $f_{1}, f_{2}, f_{3}$ et $f_{4}$ sont des paramètres sans dimension. $f_{1}$ rend compte de la variation de la photosynthèse en fonction de l'éclairement. C'est une fonction homographique déduite des observations de HESKETH \& MUSGRAVE (1962). $\mathrm{f}_{2}$ traduit les modifications de la photosynthèse provoquées par les variations de la teneur en glucides de réserve suivant une formulation empirique inchangée depuis la première version du modèle (CHILDS et al., 1977). Le pourcentage de feuilles éclairées $\mathrm{f}_{3}$ dépend de l'indice foliaire total de la culture et de l'angle que fait le soleil avec l'horizontale. Sa formulation est déduite des travaux de 
LEMEUR \& BLAD (1974). $f_{4}$ est une fonction du troisième degré du potentiel hydrique foliaire $h_{\mathrm{f}}$ qui, dans sa forme initiale, et pour des potentiels compris entre $-0,407 \cdot 10^{4}$ et $-0,163 \cdot 10^{5} \mathrm{~cm}$ d'eau, traduit la réduction de la photosynthèse provoquée par la fermeture hydroactive des stomates telle que l'observe expérimentalement BOYER (1970).

\section{La respiration horaire}

La respiration totale $R_{t}$ est estimée, par une relation linéaire empirique, en fonction de la température et du stock de glucides de réserve G. Suivant une décomposition proposée par MC CREE (1970), la respiration totale est affectée à l'entretien et à la croissance de la plante. La respiration d'entretien $R_{m}$ varie avec la température suivant l'équation de MC CREE (1974). La respiration de croissance $R_{c}$ correspond à la quantité de respiration disponible après satisfaction de l'entretien et permet d'estimer l'accroissement de matière sèche structurale. La quantité d'assimilats primaires $\mathrm{S}$ destinée à être transformée en fraction structurale est calculée en supposant que $1 \mathrm{mg} \mathrm{C} \mathrm{CH}_{2} \mathrm{O}$ perdu par respiration de croissance correspond à l'élaboration de $3,76 \mathrm{mg} \mathrm{CH} \mathrm{CH}_{2} \mathrm{O}$ de structure, soit une efficience de la croissance $Y_{c}$ de 0,79 .

\section{La production végétale}

La période de croissance est décomposée en 2 stades de développement : de la levée à la floraison et de la floraison à la maturité physiologique. Ces stades sont définis à partir des sommes de température. La croissance débute lorsque la somme des températures, dépassant un certain seuil, atteint un total caractéristique de la variété. L'accroissement journalier de la fraction structurale, converti en masse de matière sèche $\left(1,05 \mathrm{~g} \mathrm{CH}_{2} \mathrm{O} \cdot \mathrm{g}^{-1}\right.$ MS d'après TSCHESCHKE \& GILLEY, 1979) est réparti en quatre compartiments : les feuilles, les tiges, les racines et les épis. 10 p. 100 de la fraction structurale sont systématiquement attribués au système racinaire quel que soit le stade de développement du maïs. Le reste, 90 p. 100 de la fraction structurale, est distribué aux feuilles et aux tiges avant la floraison (le rapport de la croissance des feuilles $\Delta \mathbf{B M}_{\mathrm{f}}$ à la croissance totale de la partie épigée $\Delta \mathrm{BM}_{\mathrm{t}}$ variant linéairement de 1 à la levée à 0 à la floraison), puis est affecté à la croissance des épis après la floraison.

L'accroissement, par unité de surface de sol, de la masse de feuilles de la culture est :

$$
\Delta \mathrm{BM}_{\mathrm{f}}^{\prime}=\Delta \mathrm{BM}_{\mathrm{f}} \cdot \mathrm{iF}
$$

où $\Delta \mathrm{BM}_{\mathrm{f}}$ est exprimé en $\mathrm{g}$ de matière sèche par unité de surface foliaire.

L'accroissement correspondant de la surface foliaire est :

$$
\Delta \mathrm{iF}=\Delta \mathrm{BM}_{\mathrm{f}}^{\prime} \cdot \mathrm{k}
$$

avec $\mathrm{k}$ la surface massique foliaire en $\mathrm{dm}^{2}$ feuille. $\mathrm{g}^{-1} \mathrm{MS}$.

Notons que la masse de grain, exprimée en quintaux par hectare, est déduite de la masse des épis en faisant l'hypothèse d'un poids de grain égal à 85 p. 100 du poids de l'épi.

\section{Le développement du système racinaire}

Il n'y a pas de couplage entre la dynamique des racines et l'affectation d'une partie de la fraction structurale au système racinaire. A partir de la levée, le modèle rend compte d'un accroissement linéaire de la profondeur racinaire pendant un nombre de jours fixé. La profondeur à la levée est considérée égale à $15 \mathrm{~cm}$. La croissance du système racinaire se termine à la floraison mâle. La profondeur maximale atteinte $z^{*}$ est un paramètre d'entrée du modèle ; elle dépend du type de sol, du génotype et des conditions climatiques pendant la saison de croissance. La répartition relative des racines est déduite des observations de GRIME $e t$ al. (1975).

$$
\begin{aligned}
\operatorname{RDF}(z)=2,0633-3,244\left(\frac{Z}{Z^{*}}\right) & + \\
& +1,676\left(\frac{Z}{Z^{*}}\right)^{2} .
\end{aligned}
$$

\section{Mise en ouvre de CORNGRo}

On trouvera sur le tableau 1 les paramètres et variables d'entrée du modèle. Parmi les paramètres on distingue ceux que l'utilisateur devra estimer et proposer avant chaque simulation et ceux qui sont supposés connus et constants pour toutes les simulations. On y indique aussi les coefficients qui sont identifiés par calage.

D'un point de vue pratique le programme de calcul de CORNGRO comporte 1200 lignes de programme

TABLEAU 1

- Liste des variables d'entrée et des paramètres de CORNGRO

- List of input variables and parameters of CORNGRO.

Variables d'entrée :

- Rayonnement global journalier (W. $\left.\mathrm{m}^{2}\right)$.

- Températures extrêmes journalières $\left({ }^{\circ} \mathrm{C}\right)$.

- ETP journalier $(\mathrm{mm})$.

- Précipitation journalière $(\mathrm{mm})$.

- Dose d'irrigation journalière $(\mathrm{mm})$.

Paramètres à estimer :

- Caractéristiques hydriques du sol,

- relation potentiel matriciel - teneur en eau,

- relation conductivité hydraulique - teneur en eau,

- profil de teneur en eau du sol en début de simulation.

- Caractéristiques du végétal,

- profondeur racinaire maximale,

- distance moyenne entre racines,

- sommes de température du semis à :

- la floraison.

- la maturité.

Paramètres supposés connus :

- Résistance hydraulique des parties aériennes de la plante $\mathbf{r}_{\mathrm{x}}$.

- Potentiel hydrique foliaire minimal $h_{\mathrm{f}}^{*}$.

Coefficients de calage:

- Po photosynthèse brute du couvert au point de saturation de la lumière $\mathrm{W} \cdot \mathrm{m}^{-2}$ )

- $\mathrm{k}$ surface massique foliaire $\left(\mathrm{dm}^{-}, \mathrm{g}^{-1}\right)$. 
en FORTRAN IV. Le temps d'exécution de la simulation d'une saison de culture complète est d'environ 20 s CPU sur un I.B.M. 3081 D.

Bien que CORNGRO simule les dynamiques hydriques et végétales à des pas de temps horaires, la structure du modèle et la nature des variables d'entrée font que les résultats de simulation ne sont physiquement et biologiquement cohérents que s'ils sont intégrés sur des pas de temps égaux ou supérieurs à la journée. En effet, certaines variables d'entrée sont insuffisamment définies afin de simuler des processus horaires : il en est ainsi des précipitations et de l'ETP dont les stations météorologiques ne fournissent pas l'intensité pour la première et ne proposent qu'une estimation moyenne sur 10 jours pour la seconde. De plus, certains processus physiques, décrits par le modèle sont simplifiés par des hypothèses peu valides à des pas de temps inférieurs à la journée : les transferts hydriques sont supposés isothermes et les flux d'eau dans le végétal sont supposés conservatifs.

\section{ESTIMATION DES COEFFICIENTS DE CALAGE}

Pour les auteurs de CORNGRO, un calage du modèle est indispensable pour tout site ou toute variété nouvelle. Ce calage ne concerne pas le sousmodèle des transferts hydriques dont les paramètres sont soit connus, soit mesurables expérimentalement. Du fait de l'empirisme de certaines relations du sousmodèle de croissance du maïs, ce dernier nécessite, pour chaque site ou variété de maïs nouveaux, un réajustement à une situation expérimentale connue. Pour réaliser l'ajustement CHILDS et al. (1977) ont été amenés à faire varier les valeurs de 2 coefficients Po et $\mathbf{k}$ (cf. tabl. 1) ; le choix de ces coefficients de calage est motivé par le fait qu'ils sont difficiles à mesurer, mais surtout parce qu'ils permettent un ajustement efficace du modèle. Po et $k$ sont identifiés dans des situations pour lesquelles le rendement de la culture et sa surface foliaire sont connus, ils sont supposés invariants à site et variété constants, et ceci pour des conditions climatiques variables. C'est précisément ce point que nous tentons de vérifier ici en recherchant s'il existe un même couple (Po, k), capable de rendre compte du rendement et de la surface foliaire lors de 2 années climatiquement contrastées.

\section{A. Position du problème}

Soit $\mathrm{f}$ la fonction implicite rendant compte du rendement $\rho$ et de la surface foliaire SF en fonction de la variété $\mathrm{v}$, des caractéristiques du sol $\mathrm{s}$, du climat $\mathrm{t}$ et du couple (Po, k), nous avons :

$$
(\rho, S F)=f(v, s, t, P o, k)
$$

Pour 2 années à climat contrasté, $t_{1}$ et $t_{2}$, nous recherchons les couples $(\mathrm{Po}, \mathrm{k})$ qui sont à la fois solution de :

$$
(\mathrm{Po}, \mathrm{k})=\mathrm{f}^{-1}\left(\mathrm{v}, \mathrm{s}, \mathrm{t}_{1}, \mathrm{p}_{1}, \mathrm{SF}_{1}\right)
$$

et de

$$
(\mathrm{Po}, \mathrm{k})=\mathrm{f}^{-1}\left(\mathrm{v}, \mathrm{s}, \mathrm{t}_{2}, \mathrm{\rho}_{2}, \mathrm{SF}_{2}\right)
$$

compte tenu de la complexité de $f$, la recherche d'une solution analytique n'est pas envisageable. En conséquence, nous recherchons géométriquement l'intersection des 2 ensembles de couples ( $\mathrm{Po}, \mathrm{k})$, solutions respectives des équations (9) et (10).

Nous avons pris pour références climatiques sèches et humides les années 1976 et 1981 en Savoie, région dans laquelle l'irrigation est faiblement développée et n'élimine donc pas l'effet d'un déficit hydrique climatique. Ce dernier, presque négligeable en 1981, dépasse $200 \mathrm{~mm}$ en 1976 et se manifeste nettement lors de la floraison mâle du maïs (période de grande sensibilité à la sécheresse) située pendant la deuxième quinzaine de juillet.

Ne disposant pas de déterminations expérimentales de $\rho$ et SF en une parcelle donnée pour les 2 années considérées, nous avons plutôt recherché, pour cette région, les intervalles de variation des rendements et surfaces foliaires de maïs en 1976 et 1981 (cf. tabl. 2).

\section{TABLEAU 2}

Intervalles de variation observés pour les 2 années humide et sèche, du rendement et de la surface foliaire.

Range values of observed yield and leaf area in wet and dry years.

\begin{tabular}{ccc}
\hline \hline Année & $\begin{array}{c}\text { Rendement } \rho \\
\left(\mathrm{q} \cdot \mathrm{ha}^{-1}\right)\end{array}$ & $\begin{array}{c}\text { Surface foliaire SF } \\
\left(\mathrm{dm}^{2} \cdot \text { pied }^{-1}\right)\end{array}$ \\
\hline 1981 & $70-110$ & $35-40$ \\
1976 & $50-90$ & $30-45$ \\
\hline \hline
\end{tabular}

Notons alors que, dans la mesure où nous ne prenons plus en compte des valeurs uniques de $\rho$ et $\mathrm{SF}$, mais des domaines de valeurs, nous devons obtenir un ensemble de solutions pour Po et $\mathbf{k}$. Par cette démarche on augmente les possibilités d'existence d'un couple $(\mathrm{Po}, \mathrm{k})$ invariant entre les 2 années de simulation ; s'il n'existe pas il n'y aura " a fortiori » aucune solution du système d'équations ((9)-(10)).

Les limites des intervalles de variation de $\rho$ et SF ont été déterminées à partir d'une enquête effectuée par CHEvRIER (1981) à La Balme (Savoie). Ils traduisent la diversité des situations pédologiques, variétales et culturales présentes sur le lieu d'enquête et incluent des parcelles comparables d'une année à l'autre et qui possèdent les caractéristiques retenues pour les simulations (tabl. 3). En ce qui concerne les paramètres pédo-

\section{TABLEAU 3}

Valeurs de référence de quelques paramètres du modèle. Ces paramètres conservent, sauf indications contraires, pour l'ensemble des simulations, des valeurs constantes.

Reference values of some input parameters.

\begin{tabular}{lc}
\hline \multicolumn{1}{c}{ Paramètre } & Valeur \\
\hline Profil initial de teneur en eau & Capacité au champ \\
Potentiel de pression à la base du profil de sol & $\mathrm{h}=0$ \\
Variété de maïs & $\mathrm{LG} 11$ \\
Potentiel foliaire critique $\mathrm{h}_{\mathrm{f}}^{*}$ & $-16,3 \times 10^{5} \mathrm{~cm} \mathrm{H} \mathrm{H}_{2} \mathrm{O}$ \\
Profondeur racinaire maximale $\mathrm{z}^{*}$ & $100 \mathrm{~cm}$ \\
Somme des températures semis-floraison & $790^{\circ} \mathrm{C} . \mathrm{j}$ \\
Somme des températures semis-maturité & $1600^{\circ} \mathrm{C} . \mathrm{j}^{-1}$ \\
Densité du peuplement & 80000 pieds.ha $^{-1}$ \\
\hline
\end{tabular}


logiques choisis, ils sont caractéristiques d'un sol de texture argilo-limoneuse. Lors des analyses de sensibilité, nous envisagerons également l'effet d'une modification texturale avec un sol sablo-limoneux. Les caractéristiques hydrodynamiques (fig. 2 et 3 ) de ces sols sont issues de VAN BAVEL \& LASCANO (1979) et BRUCE (1972).
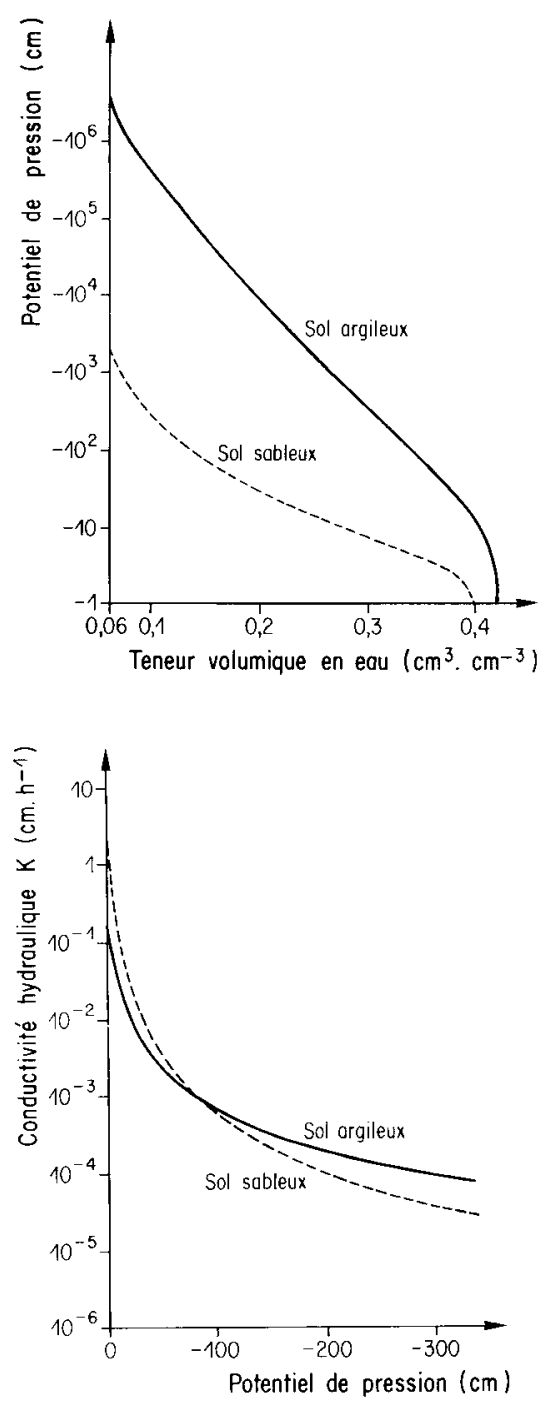

Fig. 2 et 3

Courbes de rétention et relations potentiel de pression-conductivité hydraulique utilisées pour l'analyse de sensibilité.

Soil retention and soil water potentiel-hydraulic conductivity curves used in the sensitivity analysis.

\section{B. Résultats}

Pour chacune des années considérées, nous avons simulé les rendements et les surfaces foliaires correspondant à différentes valeurs de Po et $k$. Les figures 4 et 5 sont construites par l'interpolation des valeurs de $\rho$ ou SF obtenues aux nœuds d'un maillage du plan $(\overrightarrow{\mathrm{Po}}, \overrightarrow{\mathrm{k}})$. Le domaine d'exploration a été limité à des valeurs ne conduisant pas à des couples $(\rho, S F)$ aberrants. Dans les 2 années, l'augmentation de Po se traduit par une croissance simultanée du rendement et de la surface foliaire. L'influence de $\mathrm{k}$ s'exerce différemment sur $\rho$ et SF ; en effet, tandis que la surface foliaire présente un accroissement exponentiel lorsque
Année sèche
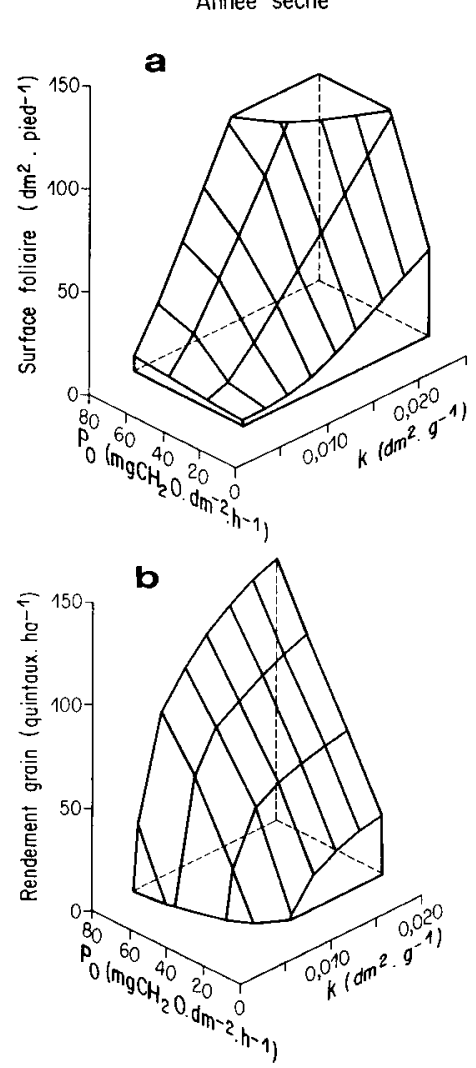

Année humide

a
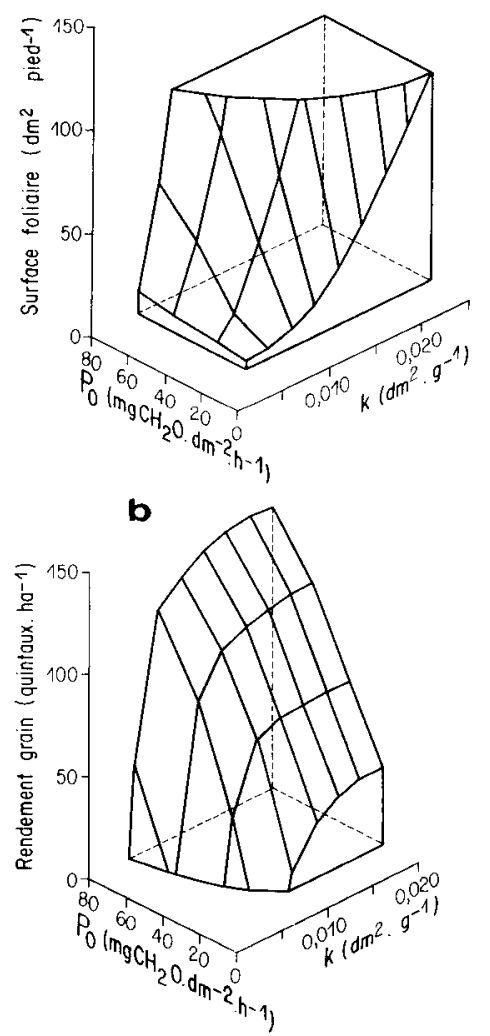

Fig. 4 et 5

Surface de réponse simulée de a) la surface foliaire $\mathrm{dm}^{2}$. pied ${ }^{-1}$ et b) du rendement, $q . h a^{-1}$ aux variations des 2 constantes de calibration. Cas d'une année sèche et d'une année humide.

Simulated response of a) leaf area $\mathrm{dm}^{2}$.plant ${ }^{-1}$ and b) yield $100 \mathrm{~kg} . \mathrm{ha}^{-1}$ to variations of the calibration coefficients in dry and wet year. 
$\mathrm{k}$ augmente, le rendement tend vers une asymptote qui est d'autant plus élevée que Po est grand. Ainsi, en année humide, lorsque $\mathrm{Po}=65$, le rendement maximal simulé est voisin de 120 quintaux de grain. Cette variation asymptotique du rendement est la conséquence d'une absorption totale du rayonnement photosynthétique actif, au-delà d'un certain indice foliaire et de la réduction de la photosynthèse par la régulation stomatique. Cette dernière est consécutive à un épuisement rapide de l'eau disponible par la transpiration de l'importante surface de feuille.

L'intersection des surfaces, représentées en figures 4 et 5 , avec les bornes des intervalles de variation de $\rho$ et SF (tabl. 2), nous donne par projection sur le plan (Po, k) les domaines représentés en figure 6. De cette manière sont définis les ensembles de couples ( $\mathrm{Po}, \mathrm{k}$ ) qui, pour une année donnée, conduisent à des simulations réalistes de $\rho$ et SF. Les figures $6 a$ et $6 b$ représentent les ensembles obtenus respectivement pour les années sèche et humide. Leur intersection est un ensemble vide (fig. 6c), ce qui signifie qu'il n'existe pas de couple (Po,k) susceptible de rendre compte à la fois des rendements et des surfaces foliaires d'une culture de maïs pour une année à déficit climatique élevé et pour une année à déficit nul. Concrètement, si l'on identifie un couple $\left(\mathrm{Po}_{\mathrm{O}} \mathrm{k}\right)$, adapté à une année humide, le modèle surestime les effets d'un déficit hydrique sur le développement de la surface foliaire, tandis que les rendements simulés restent dans l'intervalle de variation mesuré pour l'année sèche.

Ces résultats confirment et complètent ceux déjà obtenus par CHILDS et al. (1977) et TSCHESCHKE \& Gilley (1979). Cependant nos conclusions doivent

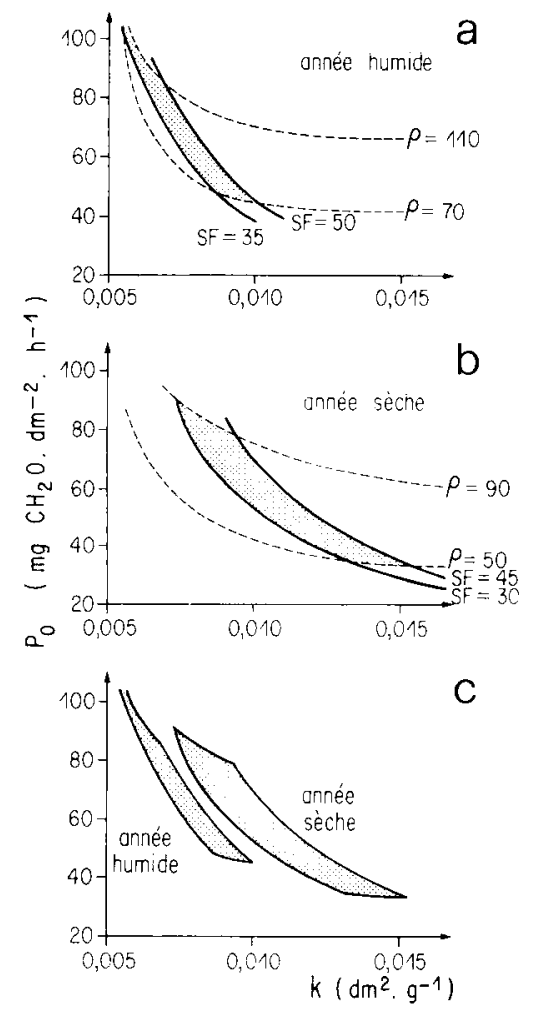

Fig. 6

Définition et comparaison des domaines de variations admissibles des constantes de calibration lors d'une année sèche et d'une année humide.

Definition and comparison of allowable variation range of the calibration constanis in dry and wet vears. être nuancées car d'une part, le choix de situations climatiques très contrastées a rendu ce test d'ajustement particulièrement exigeant pour le modèle, et d'autre part, nous avons supposé que les paramètres n'avaient pas de variations interannuelles importantes. Les aspects relatifs à l'estimation de ces paramètres seront abordés lors de l'analyse de sensibilité. Nous ne nous interesserons pas aux constantes empiriques du sousmodèle de croissance du maïs, car leurs effets sont largement effacés par le rôle prépondérant de Po et de $\mathrm{k}$. Des modifications raisonnables de ces constantes n'affectent que faiblement le rendement et la surface foliaire et conduisent à une légère déformation des surfaces disjointes de la figure $6 \mathrm{c}$, sans pour autant rendre possible l'existence d'un domaine d'intersection.

\section{Conséquences pratiques}

La structure actuelle de CORNGRO ne permet pas de simuler la croissance du maïs, en toute situation, sans la réestimation des coefficients Po et $k$. Ces coefficients sont donc ramenés au rang de simples coefficients de calage qui compensent l'inadéquation du modèle. N'est-il pas alors possible de fixer la valeur de l'un de ces deux coefficients ? A la suite de nombreux tests, TSCHESCHKE \& GILLEY (1979) proposent

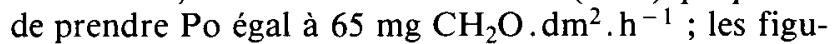
res 4 et 5 montrent que cette valeur est bien adaptée à la simulation d'une large gamme de rendements. Dans ce cas, le calage se réduit à la recherche de $\mathrm{k}$, qui joue alors le rôle d'un «super-coefficient », capable de compenser au niveau des sorties du modèle les erreurs d'estimation faites sur l'ensemble de ses paramètres, et ses insuffisances de conception.

\section{ANALYSE DE SENSIBILITÉ}

L'analyse de sensibilité consiste à évaluer les conséquences, au niveau des sorties, de variations imposées aux entrées ou aux paramètres du modèle. Le plus souvent, l'analyse de sensibilité aux variations des paramètres consiste à comparer les sorties obtenues, lorsque les $n$ paramètres ont une valeur moyenne, aux sorties obtenues lorsque chacun de ces paramètres est perturbé, tour à tour par rapport à cette valeur moyenne. En général, on effectue 2 perturbations par paramètre étudié, en l'augmentant et en le diminuant d'une quantité choisie. Dans ce cas, $2^{\text {n }}$ simulations devraient être réalisées. Le coût d'une telle analyse de sensibilité devient rapidement prohibitif lorsque la valeur de n est grande. En conséquence, nous n'avons retenu que des paramètres dont l'estimation est délicate et dont l'analyse de sensibilité n'a pas encore été réalisée par KUNDU et al. (1982) : la distance interracinaire $\Delta \mathrm{x}$, la profondeur racinaire à la maturité $\mathrm{z}^{*}$, les caractéristiques hydrodynamiques du sol, le coefficient d'extinction de l'ETP et les sommes de températures délimitant les stades de développement.

L'insensibilité du modèle à un paramètre, intervenant dans la formulation mathématique d'un processus donné, peut s'interpréter par la faible importance de ce dernier ou par sa mauvaise représentation. A l'inverse, la mise en évidence de paramètres sensibles 
doit conduire à une estimation précise de ces derniers et donc à l'amélioration du protocole expérimental de leurs mesures (voir par exemple l'analyse de sensibilité du modèle TROIKA de fonctionnement hydrique d'un plant de maïs dans LAMBERT \& REICOSKY, 1984).

Dans les analyses de sensibilité qui suivent, nous avons adopté comme valeur des coefficients : Po $=65 \mathrm{mg} \mathrm{CH}_{2} \mathrm{O} \cdot \mathrm{dm}^{2} \cdot \mathrm{h}^{-1} ; \mathrm{k}=0,0077$ et $0,0102 \mathrm{dm}^{2} \cdot \mathrm{g}^{-1}$ pour 1981 et 1976 . Les résultats des simulations correspondantes sont présentés au tableau 4, il s'agit des simulations où chaque paramètre du modèle prend sa valeur moyenne.

\section{TABLEAU 4}

Valeurs simulées en années humide et sèche de l'évapotranspiration rélle cumulée ETR et de ses composantes, évaporation du sol nu E et transpiration $T R$, du rendement en grains et de la surface foliaire. Simulated values in wet and dry years of cumulated actual evapotranspiration ETR and its components, evaporation from bare soil $E$ and transpiration $T R$, grain vield and leaf area $S F$.

\begin{tabular}{lccccc}
\hline \hline Année & $\begin{array}{c}\mathrm{E} \\
(\mathrm{mm})\end{array}$ & $\begin{array}{c}\text { TR } \\
(\mathrm{mm})\end{array}$ & $\begin{array}{c}\text { ETR } \\
(\mathrm{mm})\end{array}$ & $\rho\left(\mathrm{q} \cdot \mathrm{ha}^{-1}\right)$ & $\begin{array}{c}\text { SF } \\
\left(\mathrm{dm}^{2} \cdot \text { pied }\right.\end{array}$
\end{tabular}

\section{A. La distance interracinaire}

D'après les données de la littérature, la distance interracinaire moyenne au sein d'un horizon du profil racinaire se situerait entre 0,2 et $2 \mathrm{~cm}$ (NIMAH \& HANKS, 1973 ; MAERTENS et al., 1974 ; CHILDS et al., 1977 ; TARDIEU \& MANICHON, 1986). Aussi nous avons simulé l'effet de trois distances interracinaires $\Delta \mathrm{x}=0,2,1,0$ et $2,0 \mathrm{~cm}$, ce qui correspond à des longueurs racinaires totales de respectivement 318 , 12,7 et $3,2 \mathrm{~km}$ de racine par $\mathrm{m}^{3}$ sol, c'est-à-dire à une gamme très large de longueurs racinaires. En année humide, l'ETR cumulée de la période de croissance diminue de 1 p. 100 lorsque $\Delta x$ passe de 0,2 à $2 \mathrm{~cm}$. Il en ressort que le rendement et la surface foliaire diminuent respectivement de 4 à 3 p. 100. Dans le cas de l'année sèche, l'ETR n'est pas modifiée, la diminution du rendement négligeable, celle de la surface foliaire est identique au cas de l'année humide, $4 \mathrm{p} .100$. Un résultat analogue a été obtenu par Hillel et al. (1976) en augmentant $\Delta \mathrm{x}$ de 0,25 à $0,8 \mathrm{~cm}$.
Il semble donc que $\Delta \mathrm{x}$ soit un paramètre peu sensible et qu'il soit possible de fixer sa valeur à $1 \mathrm{~cm}$ comme dans la version originale de CORNGRO. Ce résultat soulève le problème de l'intérêt d'une prise en compte de la distance moyenne entre racines au sein du terme d'extraction racinaire ; la notion de distance moyenne est d'ailleurs contestable étant donné la répartition souvent groupée des racines (TARDIEU \& MANICHON, 1986). Le manque de réaction du modèle à $\Delta \mathrm{x}$ est cependant lié aux critères de sensibilité que nous examinons. En effet, nous n'envisageons les fluctuations des variables de sortie qu'à l'échelle de la saison de culture. Sur des intervalles de temps plus courts l'influence de $\Delta \mathrm{x}$ eût été certainement plus forte. Pour illustrer ce point, prenons le cas d'une diminution de $\mathrm{x}$ par rapport à sa valeur moyenne, cela a pour effet immédiat de diminuer la résistance sol-racine $r_{s}$, donc d'induire une augmentation des flux d'extraction racinaire et une diminution plus élevée du stock en eau de l'horizon considéré. Mais ensuite, l'extraction racinaire simulée sera inférieure à celle relative au $\Delta \mathrm{x}$ moyen, car du fait de la diminution des réserves hydriques, la conductivité hydraulique chute également et provoque une augmentation de $r_{s}$ (Eq. 3). Des mécanismes d'autocompensation à l'échelle de la saison de culture interviennent donc, qui expliquent la faible sensibilité du modèle à $\Delta \mathrm{x}$, mais aussi à la relation teneur en eau-conductivité hydraulique (KUNDU et al., 1982) et à la résistance $r_{x}$ du xylème des parties aériennes. Des simulations effectuées, et non représentées dans ce développement, en modifiant $r_{x}$ de $2500 \mathrm{~h}$ à $7500 \mathrm{~h}$, confirment ce résultat.

\section{B. La profondeur racinaire à maturité}

Le tableau 5 rend compte des modifications du bilan hydrique simulé, provoquées par le passage de $z^{*}$ de 100 à $150 \mathrm{~cm}$. On note tant pour l'année humide que pour l'année sèche, une augmentation de l'évapotranspiration réelle, des remontées capillaires, des surfaces foliaires finales et du rendement du maïs dans le cas d'un système racinaire profond. Ces augmentations sont néanmoins beaucoup plus sensibles lors de l'année sèche, où l'accroissement de la réserve en eau consécutif à un approfondissement du système racinaire permet au maïs d'effectuer son cycle sans contraintes. En conséquence, il profite au mieux des conditions d'ensoleillement favorables de 1976 et accroît son rendement. Dans ce cas la profondeur racinaire

TABLEAU 5

Analyse de sensibilité de CORNGRO aux variations de la profondeur d'enracinement.

Sensitivity in wet and dry years of predicted values of evapotranspiration, grain yield and leaf area variations in root depth.

\begin{tabular}{ccccccc}
\hline \hline Année & $\begin{array}{c}\text { Profondeur } \\
\text { d'enracinement } \\
(\mathrm{cm})\end{array}$ & $\mathrm{E}(\mathrm{mm})$ & $\mathrm{TR}(\mathrm{mm})$ & $\mathrm{R}_{\mathrm{c}}(\mathrm{mm})$ & $\rho\left(\mathrm{q} \cdot \mathrm{ha}^{-1}\right)$ & $\mathrm{SF}\left(\mathrm{dm} \mathbf{m}^{2} \mathrm{pied}{ }^{-1}\right)$ \\
\hline \multirow{3}{*}{ humide } & 100 & 105 & 240 & 6 & 92,4 & 42,9 \\
& 150 & 111 & 242 & 25 & 95,3 & 43,6 \\
\hline \multirow{2}{*}{ sèche } & 100 & 35 & 225 & 13 & 80,3 & 41,5 \\
& 150 & 32 & 296 & 31 & 106,5 & 53,3 \\
\hline \hline
\end{tabular}


est un paramètre sensible car elle détermine soit une contrainte hydrique importante $\left(\mathrm{z}^{*}=100 \mathrm{~cm}\right)$, soit l'absence de contrainte $\left(z^{*}=150 \mathrm{~cm}\right)$. Cependant, l'estimation aussi précise soit-elle de la profondeur racinaire laisse en suspens des questions importantes. Quelles sont les interactions entre la profondeur racinaire et le régime hydrique ? Le modèle ne tient pas compte de tels mécanismes, ce qui pourrait expliquer en partie l'impossibilité de déterminer un couple ( $\mathrm{Po}$, $\mathrm{k}$, valable simultanément pour des années humide et sèche. Une profondeur racinaire en 1976, supérieure à celle de 1981 aurait permis d'aboutir à une intersection non nulle (fig. $6 c$ ) en minimisant l'effet du déficit hydrique climatique.

\section{La texture du sol}

Le changement de texture modifie les courbes de rétention et de conductivité. La sensibilité de CORNGRO à cette double modification, pour le cas considéré et toutes choses égales par ailleurs, est faible.

Ainsi, en année humide, les quantités d'eau consommées sont très proches pour les sols argileux et sableux : respectivement 240 et $241 \mathrm{~mm}$ pour la transpiration, 105 et $110 \mathrm{~mm}$ pour l'évaporation. Les flux en profondeur sont de directions opposées. La remontée capillaire est de $6 \mathrm{~mm}$ pour le sol argileux. Le drainage atteint $27 \mathrm{~mm}$ pour le sol sableux qui présente à la fin de la saison de croissance un déficit hydrique de l'ordre de $70 \mathrm{~mm}$. Les productions de grains atteignent 92,4 et 95,0 quintaux. ha ${ }^{-1}$. Les surfaces foliaires sont pratiquement identiques : 42,9 et 43,0 $\mathrm{dm}^{2}$ feuille. pied $^{-1}$ (fig. 7).

En année sèche, la dynamique des flux en profon-

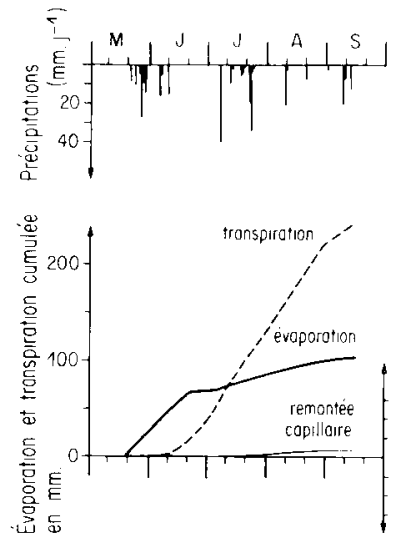

Sol argileux
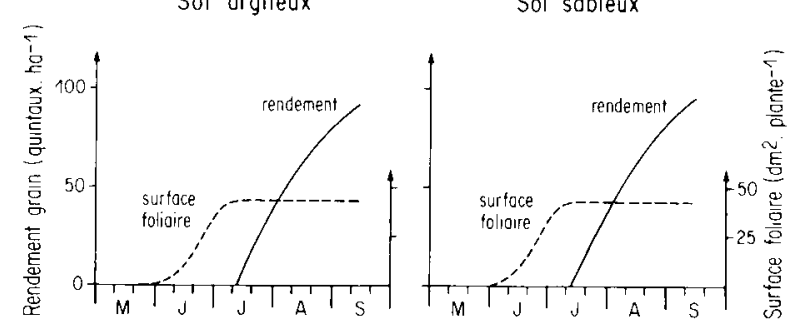

Fig. 7

Evolution simulée en année humide des principaux flux hydriques, du rendement et de la surface foliaire : cas d'un sol sableux et d'un sol argileux.

Predicted changes, in a wet year, of the main water fluxes, grain yield and leaf area : the cases of sandy and clayey soil. deur est la même que celle de l'année humide : une remontée capillaire de $12 \mathrm{~mm}$ pour le sol argileux, un drainage de $6 \mathrm{~mm}$ pour le sol sableux. L'évaporation du sol nu est faible, 35 et $46 \mathrm{~mm}$ respectivement. La transpiration atteint $225 \mathrm{~mm}$ pour le sol argileux et $263 \mathrm{~mm}$ pour le sol sableux. La baisse de production de grain est de 13 p. 100 pour le sol argileux et 21 p. 100 pour le sol sableux, celle de la surface foliaire 3 et 2 p. 100.

Les résultats montrent l'existence d'une non-proportionnalité entre production végétale et transpiration ou entre production végétale et variation de stock lorsque l'on passe du sol argileux au sol sableux. Ce point s'explique par la présence dans CORNGRO d'un facteur $\mathrm{f}^{4}$ limitant la photosynthèse sous l'action de potentiels hydriques foliaires faibles (éq. 5 et annexe 1). Ces derniers diminuent notamment lorsque les forces de rétention en eau du sol sont élevées. Or, les variations de stock et les transpirations cumulées n'expriment pas la variabilité temporelle des potentiels de rétention de l'eau extraite par la plante, d'où l'existence de non-proportionnalités.

Dans leur analyse de sensibilité KUNDU et al. (1982) modifient indépendamment courbe de rétention et courbe de conductivité en multipliant, dans un premier temps, le potentiel de pression par un scalaire variant de 0,01 à 100 , puis la conductivité de la même manière, sans tenir compte d'une quelconque liaison fonctionnelle entre ces grandeurs caractéristiques. Dans le cas du potentiel de pression, ils observent une sensibilité assez faible du rendement et de la surface foliaire lorsque le scalaire varie entre 0,01 et 1 . Par contre, $\rho$ et SF diminuent rapidement lorsqu'il passe de 1 à 100. Dans le cas de la conductivité hydraulique, la sensibilité de $\rho$ et SF est nettement moins importante. La gamme 1 à 100 reste cependant la zone la plus sensible. Les résultats ont des conséquences sur la précision à rechercher dans la détermination des courbes caractéristiques. Un doublement (ou une division par 2) du potentiel de pression provoque une diminution (ou une augmentation) du rendement et de la surface foliaire qui peuvent atteindre 10 quintaux. ha ${ }^{-1}$ et $5 \mathrm{dm}^{2}$ feuille.pied ${ }^{-1}$. En pratique, on s'attachera à disposer d'une courbe de rétention précise. Une estimation assez grossière de la conductivité hydraulique est par contre suffisante.

\section{Le coefficient d'extinction de l'ETP}

La valeur retenue dans CORNGRO du coefficient $\beta$ (Eq. 1) est de 0,438. Elle semble cependant susceptible de varier puisque UCHIJIMA (1976) constate à partir de résultats expérimentaux un intervalle de variation de $\beta$ allant de 0,450 à 0,650 . L'analyse de la sensibilité du modèle aux variations de $\beta$ est donc réalisée suivant l'intervalle le plus large.

Quelle que soit l'année de référence, humide ou sèche, le passage de 0,438 à 0,650 se traduit par une diminution de l'ETR, de la surface foliaire et du rendement. Pour l'année humide, ces diminutions sont respectivement de 6 p. 100,4 p. 100 et 31 p. 100 ; pour l'année sèche elles atteignent 10 p. 100, 65 p. 100 et 41 p. 100. Si la décroissance de l'ETR est relativement faible, la part de l'évaporation du sol nu passe de 30 à 37 p. 100 en année humide et de 13 à 25 p. 
100 en année sèche. Cette augmentation est provoquée par la diminution de surface foliaire. Ce résultat semble en première approximation assez surprenant, car à même surface foliaire, la variation de $\beta$ de 0,438 à 0,650 entraîne une augmentation de la transpiration potentielle. Pour répondre à cette demande climatique, la plante simulée puise plus dans les réserves hydriques du sol et développe des potentiels hydriques foliaires fortement négatifs. Ceci a un effet dépressif sur la photosynthèse : (cf. facteur $\mathrm{f}^{4}$ dans l'équation (5)) et par conséquence sur l'accroissement de surface foliaire. Ainsi dans l'équation (1), par une augmentation $\operatorname{de} \beta$, on aboutit à une diminution de l'indice foliaire IF simulé et du produit $\beta$. IF.

Le coefficient d'extinction $\beta$ apparaît donc être un paramètre sensible, néanmoins une mauvaise détermination expérimentale peut passer inaperçue lors du calage. Ainsi en posant $\beta=0,650$ il est possible de déterminer $\mathrm{k}$, de manière à conserver à l'ETR, au rendement et à la surface foliaire des valeurs proches de celles obtenues avec $\beta=0,438$; nous obtenons ces résultats avec $\mathrm{k}=0,0090$ en année humide et $\mathrm{k}=$ 0,0162 en année sèche.

\section{E. La somme des températures}

La période de développement du maïs, du semis à sa maturité, est divisée, dans CORNGRO, en deux stades physiologiques successifs : la croissance de l'appareil végétatif et la production des épis. Ces deux stades sont délimités à l'aide de la somme des températures moyennes journalières de l'air au-dessus d'un certain seuil. CORNGRO qui dans sa version originale utilise un seuil de $10^{\circ} \mathrm{C}$ a été modifié pour le seuil de $6^{\circ} \mathrm{C}$ adapté au cadre climatique et aux références françaises.

L'incertitude sur les sommes de température nécessaires à la réalisation des différents stades a déjà été estimée par plusieurs auteurs. DUBURCQ et al. (1983) montrent par des essais réalisés en un seul lieu, sur plusieurs variétés et conditions de milieu, que la variabilité des sommes de température s'étale de 1 à 2 p. 100 pour une variété donnée.

Cette estimation apparaît toutefois très inférieure à celle obtenue par BLOC \& GOUET (1977), qui à partir d'expérimentations menées en plusieurs régions climatiques françaises, aboutissent à une variabilité d'environ 10 p. 100 pour une même variété. Sans avoir la possibilité de trancher entre ces deux résultats divergents, nous avons testé l'impact de l'incertitude maximum pour la variété LG11, dont les caractéristiques figurent dans le tableau 3.

Pour l'année humide, le raccourcissement de la période végétative, consécutif à une diminution des seuils des sommes de températures $\mathrm{m}$ de 10 p. 100 (m -10 p. 100$)$, réduit la consommation en eau $(E T R=304 \mathrm{~mm})$ et affecte le rendement et la surface foliaire de -20 p. 100 et -34 p. 100 . Dans le cas opposé $(\mathrm{m}+10$ p. 100), l'ETR atteint $390 \mathrm{~mm}$, le rendement et la surface foliaire augmentent de 13 p. 100 et 37 p. 100 . Dans ces deux cas, l'évaporation du sol nu reste voisine de celle du cas moyen $E=105 \mathrm{~mm}$. Si nous modifions $\mathrm{k}$ pour maintenir $\beta$ et $\mathrm{SF}$ à des valeurs proches de la moyenne, des résultats comparables sont obtenus en utilisant $\mathrm{k}=0,0085$ pour $m-10$ p. 100 et $k=0,0070$ pour $m+10$ p. 100 soit respectivement une augmentation et une diminution de $\mathrm{k}$ d'environ 10 p. 100.

\section{CONCLUSION}

Le modèle CORNGRO associe la dynamique des transferts hydriques dans le système sol-plante à celle de la croissance d'une culture de maïs. Le support conceptuel du sous-modèle des transferts d'eau est une analogie électrique : l'eau circule des points dont le potentiel hydrique est élevé vers les points à faible potentiel, des résistances fixes ou variables s'opposent à cette circulation. Ce sous-modèle ne comporte pas de coefficient de calage, mais uniquement des paramètres parfaitement définis dont la détermination expérimentale peut être délicate. Le sous-modèle relatif à la croissance de la culture fait intervenir deux coefficients de calage. Dans cette note nous avons cherché à définir les principes et les domaines d'utilisation de CORNGRO en étudiant d'une part le rôle des coefficients de calage et, d'autre part, la sensibilité du modèle à ses paramètres.

En ce qui concerne les coefficients de calage, le premier d'entre eux, Po, intervient dans le calcul de la photosynthèse d'une population de feuilles. Il ne représente plus directement le taux de photosynthèse brute au point de saturation à la lumière car nous ne pouvons appliquer, sans adaptation, au niveau d'un couvert, une relation décrivant un processus intervenant au niveau de la feuille (O'NEIL, 1979). N'ayant plus un sens physique direct et compte tenu de la sensibilité des sorties du modèle - surface foliaire et rendement en grain - à ses variations, Po peut être fixé «a priori ». Pour le second coefficient, k, l'analyse de sensibilité et les tests de validation montrent qu'il ne peut être fixé. Il semble devoir être réestimé à chaque simulation, d'une année à l'autre notamment. Cela signifie qu'il sera nécessaire lors d'une application quelconque de disposer de valeurs mesurées du rendement et de la surface foliaire, afin de procéder à son identification. La prédiction du rendement semble donc impossible avec CORNGRO. Les résultats obtenus par CHILDS et al. (1977) et TSCHESCHKE \& GILLEY (1979) montrent cependant que $\mathrm{k}$ peut être maintenu constant si le modèle reste utilisé avec des conditions climatiques et édaphiques proches de celles du calage, mais avec des conditions d'alimentation en eau de la culture différentes.

L'analyse de sensibilité a permis de différencier les paramètres du modèle suivant leur influence. On distingue deux cas :

- CORNGRO s'avère relativement insensible aux variations de certains paramètres, tels que la distance interracinaire moyenne sur le profil de sol, la résistance hydraulique du xylème et la relation conductivité hydraulique-teneur en eau. Une estimation précise de ces derniers n'est donc nullement utile dans l'état actuel du modèle. On note en particulier que le terme d'extraction racinaire de CORNGRO, de structure très classique, ne valorise pas une prise en compte détaillée de la géométrie du système racinaire.

- Les variations de la profondeur racinaire maximum, de la relation potentiel matriciel-teneur en eau, des sommes de températures et du coefficient d'extinc- 
tion de l'ETP affectent significativement les simulations. Leur détermination expérimentale doit donc être conduite avec précision. Néanmoins, on a montré qu'il était possible pour différentes valeurs d'un même paramètre, d'ajuster $\mathrm{k}$ de manière à conserver au rendement, à la surface foliaire finale et aux termes du bilan hydrique cumulé, des valeurs quasi constantes. Aussi le coefficient de calage, $k$, compense la pauvreté de représentation de certains processus, mais aussi les incertitudes d'évaluation des paramètres. Cela a pour conséquence de remettre en cause la valeur identifiée de $\mathrm{k}$, si l'on fait varier, de la simulation de calage à une autre simulation, la valeur d'un ou plusieurs des paramètres, dits « sensibles ".

En définitive, il apparaît d'après les résultats de cette étude que le domaine d'utilisation de CORNGRO se circonscrit aux situations où l'on dispose d'une estimation du rendement et de la surface foliaire de la culture du maïs. Sous réserves de validations expérimentales, le modèle permet la simulation des processus intermédiaires, tant au niveau du développement de la culture que de l'évolution du bilan hydrique.

L'utilisation de CORNGRO peut alors être envisagée soit pour alléger des dispositifs de suivi des transferts sous culture, soit pour simuler des grandeurs peu accessibles à la mesure, telles que les flux de drainage ou de remontée capillaire. L'illustration d'une telle application sera abordée dans un autre article.

La prédiction du rendement en fonction des contraintes hydriques semble toutefois possible lors de la comparaison en un même lieu de différentes politiques d'irrigation (TSCHESCHKE \& GILLEY, 1979); ceci devrait permettre de mettre en évidence le rôle de la distribution temporelle des apports d'eau par le biais de simulations exploratoires.

\section{REMERCIEMENTS}

Nous tenons à remercier M. GILley, Professeur à l'Université du Nebraska (Etats-Unis), pour avoir aimablement mis à notre disposition une copie du modèle CORNGRO.

Reçu le 9 juillet 1985. Accepté le 10 juin 1987.

\section{ANNEXE 1}

Expressions mathématiques des facteurs limitants de la photosynthèse :

\section{APPENDIX 1}

Mathematical expressions of the limiting factors of photosynthesis :

$$
\begin{aligned}
\mathrm{f}_{1}= & \frac{3,39 \cdot 10^{-3} \mathrm{R}}{1+2,37 \cdot 10^{-3} \mathrm{R}} \\
\mathrm{f}_{2}= & \frac{84,5-\mathrm{G}}{84,5} \\
\mathrm{f}_{3}= & \frac{\left(1-\mathrm{e}^{\alpha \cdot \mathrm{iF}}\right)}{\alpha \cdot \mathrm{iF}} \\
\mathrm{f}_{4}= & 0,74 \cdot 10^{-2} \mathrm{~h}_{\mathrm{f}}^{3}+0,149 \cdot 10^{-7} \mathrm{~h}_{\mathrm{f}}^{2}+ \\
& +0,127 \cdot 10^{-3} \mathrm{~h}_{\mathrm{f}}+1,32
\end{aligned}
$$

avec $\mathbf{R}$ : irradiation globale (W. $\mathrm{m}^{-2}$ ).

$\mathrm{G}$ : réserve glucidique de la plante $\left(\mathrm{mg} \mathrm{CH}_{2} \mathrm{O} \cdot \mathrm{dm}^{-2}\right.$ feuille).

$h_{\mathrm{f}}$ : potentiel foliaire $(\mathrm{cm})$.

$\mathrm{iF}$ : indice foliaire.

$\alpha$ : coefficient d'extinction du rayonnement.

\section{ANNEXE 2}

\section{Equations réglant la respiration horaire.} Equation de Mc CREE (1974).

\section{APPENDIX 2}

Equations of hourly respiration. The MCCREE equation (1974).

$$
\mathrm{R}_{\mathrm{m}}=\mathrm{C}_{30}\left(0,044+0,0019 \mathrm{~T}+0,0010 \mathrm{~T}^{2}\right) . \mathrm{S} .
$$

$\mathrm{R}_{\mathrm{m}}$ : respiration de maintenance ou d'entretien $\left(\mathrm{mg} \mathrm{CH}_{2} \mathrm{O} \cdot \mathrm{dm}^{-2}\right.$ feuille. $h^{-1}$ ).

$\mathrm{T}$ : la température moyenne horaire en ${ }^{\circ} \mathrm{C}$.

$\mathrm{S}$ : masse de la fraction structurale rapportée à un $\mathrm{dm}^{2}$ de feuille (g. dm $\left.{ }^{-2}\right)$.

Le coefficient $\mathrm{C}_{30}$ déduit des travaux de Penning de VRIes (1972) est égal à $0,75 \mathrm{mg} \mathrm{CH} \mathrm{H}_{2} \mathrm{O} \cdot \mathrm{h}^{-1}$ par $\mathrm{g}$ de fraction structurale.

$$
R_{t}=(0,0039+0,0038 \mathrm{~T}) \mathrm{G}
$$

$R_{t}$ respiration totale, $G$ stock de glucides de réserve

$$
\mathrm{R}_{\mathrm{c}}=\mathrm{R}_{\mathrm{t}}-\mathrm{R}_{\mathrm{m}}
$$

avec $R_{c}$ la respiration de croissance.

La quantité d'assimilats primaires transformée en fraction structurale s'écrit :

$$
\Delta S=\frac{Y_{c}}{1-Y c} \cdot R_{c}
$$

avec $Y_{c}$ l'efficience de la croissance de la fraction structurale.

\section{RÉFÉRENCES BIBLIOGRAPHIQUES}

Baker C. H., Horrocks R. D., 1976. CORNMOD, a dynamic simulator of corn production. Agric. Systems, 4, 57-77.

Van Bavel CHM., Lascano R., 1979. CONSERB : A numerical method to compute soil water content and temperature profiles under a bare surface. Texas A. $\S$ M. Univ., Dept. Soil Crop Sci. : $75 \mathrm{p}$.

Bloc D., Gouet J. P., 1977. Influence des sommes de température sur la floraison et la maturité du mais. Ann. Amelior. Plantes, 28, $89-111$.

Boyer J. S., 1970. Leaf enlargement and metabolic rates in corn, soybean and sunflower at various leaf water potentials. Plant Physiol., 46, 233-235.

Bruce R. R., 1972. Hydraulic conductivity evaluation of the soil profile from soil water retention relations. Soil Sci. Soc. Am. Proc., $36,555-561$.

Chevrier L., 1981. Contribution à l'étude du fonctionnement hydrique des sols à nappe de la haute vallée du Rhône. Application au secteur de La Balme (Savoie). D.E.A., USTL Montpellier, 64 p.
Childs S. W., Gilley J. R., Splinter W. E., 1977. A simplified model of corn growth under moisture stress. Trans. A.S.A.E., 20, 858-865.

Curry R. B., Chen L. H., 1971. Dynamic simulation of plant growth. Part II. Incorporation of actual daily weather and partitioning of net photosynthate. Trans. A.S.A.E., 14, 1170-1174.

Duburcq J. B., Bonhomme R., Derieux M., 1983. Durée des phases végétative et reproductrice chez le maïs. Influence du génotype et du milieu. Agronomie, 3 (10) : 941-946.

Duncan W. G., 1975. SIMAIZ : A model simulating growth and yield in corn. In : D. N. Baker, P. G. Creech and F. G. Maxwell, eds. An application of system method to crop production: 32-48, Miss Agric. For. Expt. Stn., Miss. State Univ., Miss.

Feddes R. A., Kowalik P. J., Zaradny H., 1978. Simulation of field water use and crop yield. Simul. Mon., Pudoc, Wageningen, $180 \mathrm{p}$.

Garcia de Cortazar V., 1982. Modélisation de la croissance d'une culture de maïs. Thèse Docteur-Ingénieur. Université Paris-Sud, Orsay : $111 \mathrm{p}$. 
Grime D. W., Miller R. J., Willey P. C., 1975. Cotton and corn root development in two field soils of different strength characteristics. Agron. J., 67, 519.

Hesketh J. D., Musgrave R. B., 1962. Photosynthesis under field conditions: IV. Light studies with individual corn leaves. Crop Sci., 2, 311-315.

Hillel D., Talpaz H., van Keulen H., 1976. A macroscopic-scale model of water uptake by a non-uniform root system and of water and salt movement in the soil profile. Soil Sci., 121, 242-255.

Jones C. A., Kiniry J. R., (eds.) 1986. CERES-maize, a Simulation Model of Maize growth and Development. Texas A \& M University Press, College Station, U.S.A., 194 p.

Keulen van H., 1982. Crop production under semi-arid conditions as determined by moisture availability. In : Simulation of plant growth and crop production. Eds. F. W. T. Penning de Vries and H. H. van Laar : Simul. Mon., Pudoc, Wageningen : 87-89.

Kundu S. S., Skogerboe G. V., Walker W. R., 1982. Improvement and sensitivity analysis of the CORNGRO model. Ecol. Modelling, $16,209-239$.

Lambert Jr., Reicosky D. C., 1984. Dynamics of water in Zea mays L. : sensitivity analysis of TROIKA. Trans. A.S.A.E., 27, 117-124.

Lemeur R., Blad B. L., 1974. A critical review of light models for estimating the shortwave radiation regime of plant canopies. Agric. Meteorol., 14, 255-286.

Maertens C., Blanchet R., Puech J., 1974. Influence de différents régimes hydriques sur l'absorption de l'eau et des éléments minéraux pour les cultures. I. Régimes hydriques, systèmes racinaires et modalités d'alimentation en eau. Ann. Agro., 25, 575-586.

Mc Cree K. J., 1970. An equation for the rate of respiration of white clover plant grown under controlled conditions. In : Prediction and measurement of photosynthetic productivity: 221-229. Pudoc. Wageningen.

Mc Cree K. J., 1974. Equations for the rate of dark respiration of white clover and grain sorghum, as functions of dry weight, photosynthetic rate, and temperature. Crop Sci., 14, 509-514.
Nimah M. N., Hanks R. J., 1973. Model for estimating soil water and atmospheric interrelations. Soil Sci. Soc. Amer. Proc., 37, 522 532.

O'Neil R. F., 1979. Transmutations across hierarchical levels. In : Systems analysis of ecosystems, G. S. Innis \& R. V. O'Neil, eds. Statistical Ecology Series 9, 59-78.

Penning de Vries F. W. T., 1972. Respiration and growth. p. 327347. In : Reeds, Cockshull, Hand and Hurd (eds.) Crop Processes in Controlled Environments. Academic Press, London.

Rambal S., Cornet A., 1982. Simulation de l'utilisation de l'eau et de la production végétale d'une phytocénose sahélienne du Sénégal. Acta Oecologica, Oecol. Plant., 3 (17), 381-397.

Stapper M., Arkin G. F., 1980. CORNF : A dynamic growth and development model for maize (Zea mays L.). Res. Cent. Program and Model Documentation, $n^{\circ} 80-2$. Blackland Res. Cent. Temple : 91 p. Texas Agr. Exp. Stn, College Station, Texas.

Stockle C., Campbell G., 1985. A simulation model for predicting effect of water stress on yield : an example using corn. $A d v$. Irrig., 3, 283-311.

Tardieu F., Manichon H., 1986. Caractérisation en tant que capteur d'eau de l'enracinement du maïs en parcelle cultivée. I. Discussion des critères d'étude. Agronomie, 6 (4), 345-353.

Tscheschke P. D., Gilley J. R., 1979. Status and verification of Nebraska's corn growth model CORNGRO. Trans. A.S.A.E., 22, 1329-1337.

Uchijima Z., 1976. Maize and Rice. In J. L. Monteith, Ed. Vegetation and the atmosphere. Vol. 2 : 33-64, Academic Press, London.

Voltz M., 1982. Essai de prévision du rôle d'une nappe phréatique dans l'alimentation hydrique du mais. D.A.A., ENSA Montpellier, $56 \mathrm{p}$.

Wit de C. T. et al., 1978. Simulation of assimilation, respiration and transpiration of crops. Simul. Mon., Pudoc, Wageningen, $141 \mathrm{p}$. 